\title{
Oxyethylated Isononylphenols in Carbon Tetrachloride
}

\author{
Victor P. Arkhipov ${ }^{1} \cdot$ Ruslan V. Arkhipov $^{2} \cdot$ Natalia A. Kuzina $^{1} \cdot$ Andrei Filippov $^{3,4}$
}

Received: 8 July 2019 / Revised: 22 August 2019 / Published online: 1 October 2019

(c) The Author(s) 2019

\begin{abstract}
Translational diffusion coefficients of ethoxylated isononylphenol molecules $\mathrm{C}_{9} \mathrm{H}_{19} \mathrm{C}_{6} \mathrm{H}_{4} \mathrm{O}\left(\mathrm{C}_{2} \mathrm{H}_{4} \mathrm{O}\right)_{n} \mathrm{H}$ in carbon tetrachloride were measured by nuclear magnetic resonance diffusometry. The hydrodynamic radii of the molecules were determined within the framework of the Stokes-Einstein relation. We showed that ethoxylated isononylphenols in carbon tetrachloride do not form micelles, and the dependence of the diffusion coefficients and, accordingly, the hydrodynamic radii of the nonionic surfactants on the number of oxyethylene groups have a kink in the region $n=6-8$.
\end{abstract}

\section{Introduction}

Oxyethylated isononylphenols (neonols AF9-n) $\mathrm{C}_{9} \mathrm{H}_{19} \mathrm{C}_{6} \mathrm{H}_{4} \mathrm{O}\left(\mathrm{C}_{2} \mathrm{H}_{4} \mathrm{O}\right)_{n} \mathrm{H}$ are highly effective nonionic surfactants, which are used in industry [1]. Neonols are produced in a wide range of $\mathrm{n}$ values, which makes it possible to study their physicochemical properties depending on the length of the oxyethylene chain. Properties of micellar solutions of nonionic surfactants and the configuration of oxyethylene chains have been studied by optical methods [2-4], nuclear magnetic resonance (NMR) [5-7], small-angle neutron and X-ray scattering [8,9], mass spectromery [10, 11], tensometry [12], and by computer simulation [6,11, 13]. Oxyethylated nonionic surfactants demonstrate a contraction phenomenon, a change in the configuration of the oxyethylene chain from a regularly zig zag to a meander shape. Studies of $\mathrm{C}_{12}(\mathrm{EO})_{n}$ ethoxylated fatty alcohols in mild acidic aqueous solution by small-angle X-ray diffraction [9] showed that the change in the configuration of the chain from zig zag to a meander shape occurs in the range of 8-9 oxyethylene units.

Andrei Filippov

Andrei.Filippov@1tu.se

1 Department of Physics, Kazan National Research Technological University, 420015 Kazan,

Russian Federation

2 Institute of Physics, Kazan Federal University, 420008 Kazan, Russian Federation

3 Chemistry of Interfaces, Luleå University of Technology, 97187 Luleå, Sweden

4 Kazan State Medical University, 420012 Kazan, Russian Federation 
The contraction phenomenon is explained by the loss of chain stability, the formation of intramolecular hydrogen bonds between oxygen atoms, if the number of oxyethylene units $\left(\mathrm{CH}_{2}-\mathrm{CH}_{2}-\mathrm{O}\right)_{n}$ exceeds a certain critical value $[14,15]$. The phenomenon of contraction in neonols is observed [16] under the condition $n \geq 9$. Previously [17], we measured the diffusion coefficients of molecules in pure neonols AF9-n at various temperatures by NMR diffusometry. We showed that the linear dependence of the diffusion coefficients and activation energies of the translational motion of molecules of pure neonols on the number $n$ changes slope at $n \approx 9$, confirming the presence of a contraction phenomenon.

The article is devoted to the study of the phenomenon of contraction of neonol molecules in carbon tetrachloride solutions by NMR diffusometry. The choice of solvent was explained by the need to obtain true molecular solutions of neonols at relatively high concentrations. Hydrophilic and lyophilic properties of nonionic surfactant molecules lead to the formation of direct or reverse micelles in water or in hydrocarbon solvents [8, 18-22], respectively. In such systems, the molecular state of nonionic surfactants exists only at sufficiently low concentrations, lower than the critical micelle concentration (CMC). Typical CMC values of nonionic surfactants are 0.1-0.05 mM. Therefore, measurement of diffusion coefficients by NMR becomes difficult.

\section{Experimental}

Carbon tetrachloride $\mathrm{CCl}_{4}$, an aprotic, inert solvent with no acidic or basic properties, was chosen as the solvent. Molecules of carbon tetrachloride do not contain hydroxyl groups; they do not have an electric dipole moment; therefore, solutions of neonols in $\mathrm{CCl}_{4}$ are true, molecular solutions that are not prone to micelle formation. In such solutions, it is possible to study the behavior of individual nonionic surfactant molecules, and not the micelles they form. For ${ }^{1} \mathrm{H}$ NMR studies, carbon tetrachloride has another important advantage; being an aprotic solvent, it does not produce lines in the NMR spectra. True (molecular) solutions of nonionic surfactants in water or in hydrocarbon solvents exist at $\mathrm{C}<\mathrm{CMC}$, about $10^{-4} \mathrm{M}$. However, NMR measurements are difficult at such low concentrations, as they are being carried out on the verge of the sensitivity of the instruments, and, accordingly, are associated with significant experimental errors. In addition, in aqueous solutions, the formation of hydrogen bonds between the protons of water molecules and the oxygen of the hydroxyl groups of nonionic surfactants is possible, which, in turn, can affect the phenomenon of contraction.

The observed parameter is the translational diffusion coefficient of the neonol molecules, whose magnitude is determined by the viscosity of the solvent, temperature, size, and shape of the diffusant molecules (neonols). If, with an increase in the length of the oxyethylene chain of the neonols AF9-n, their zigzag shape remains, then the diffusion coefficient should change monotonously, decreasing with increasing $\mathrm{n}$. A change in the shape of the molecules as a result of contraction should, accordingly, manifest itself in the destruction of this monotony. 
The diffusion coefficients were measured using a pulsed sequence of stimulated spin echo with a pulsed gradient magnetic field on an NMR spectrometer "BrukerAvance" ( ${ }^{1} \mathrm{H}-400 \mathrm{MHz}$ ). Neonols AF9-2, AF9-3, AF9-4, AF9-6, AF9-8, AF9-9, AF9-10, and AF9-12 produced by the corporation "Nizhnekamskneftechim" [1] were studied without extra purification. For the preparation of solutions, carbon tetrachloride was used, which was dried and distilled over $\mathrm{P}_{2} \mathrm{O}_{5}$ [23]. The diffusion coefficients of the neonol molecules were measured at a fixed temperature of $20{ }^{\circ} \mathrm{C}$ in the concentration range from 100 to $0.1 \mathrm{~g} / \mathrm{L}$. The concentration ranges within which measurements of the diffusion coefficients of neonols were made are given in Table 1. Use of a homologous series of AF9-n neonols allowed us to associate the phenomenon of contraction with the length of the oxyethylene chain and changes in the nature of the translational motion of the neonol molecules.

\section{Results and Discussion}

The amplitude of the stimulated spin echo signal, according to [24], is described by the expression:

$$
A=A_{0} \exp \left(-\gamma^{2} \delta^{2} g^{2} \mathrm{D} t_{\mathrm{d}}\right)=A_{0} \exp \left(-k^{2} \mathrm{D} t_{\mathrm{d}}\right),
$$

where $\gamma$ is the hyromagnetic ratio of protons; $\delta$ and $g$ are duration and amplitude of the gradient pulse of magnetic field, respectively; $t_{\mathrm{d}}$ is the diffusion time; and $D$ is the diffusion coefficient. Measurements of diffusion coefficients were made at: $t_{\mathrm{d}}=49 \mathrm{~ms}, \delta=1 \mathrm{~ms}$, and repetition time $=5 \mathrm{~s}$. The number of scans was set from 4 to 64 depending on the amplitude of the echo signal. The number of points on the decay was kept constant, $i=16$. The maximum value of the pulse gradient was 1.27 $\mathrm{T} / \mathrm{m}$. In all solutions, a single exponential decay of the amplitude of the spin echo signal was observed, Fig. 1. This may indicate the absence of micelle formation or a high rate of exchange between molecular and micellar states of neonol molecules in solution. Figure 1 shows diffusion decays for some neonols, measured at their different concentrations in solutions.

The diffusion coefficients of neonols, Fig. 2, decrease almost linearly with an increase in their concentration in the solution, which is obviously associated with an increase in the viscosity of the solutions.

The graphs do not show nonlinearities' characteristic of transitions from the molecular to the micellar state of the nonionic surfactants in solution. Therefore, in the whole concentration range from $100 \mathrm{~g} / \mathrm{L}$ and less to $0.1 \mathrm{~g} / \mathrm{L}$, solutions of neonols in carbon tetrachloride are true molecular solutions. The weight concentration of neonols at $0.1 \mathrm{~g} / \mathrm{L}$ corresponds, on average, to a molar concentration of $0.1 \div$ $0.5 \mathrm{mM}$, and according to [18, 22, 25-27], CMC of nonionic surfactants in nonpolar, organic solvents is in the range $\approx 20 \div 200 \mathrm{mM}$.

The diffusion coefficient values of the neonol molecules under the condition of infinite dilution $D_{0}$ were determined by extrapolating the dependence of the diffusion coefficients on the concentration of neonols, Fig. 3. The dependence of $D_{0}$ on the number of oxyethylene groups in the molecules of neonols has two linear portions, with an intersection point at $n=6-8$. It can be concluded that a change 


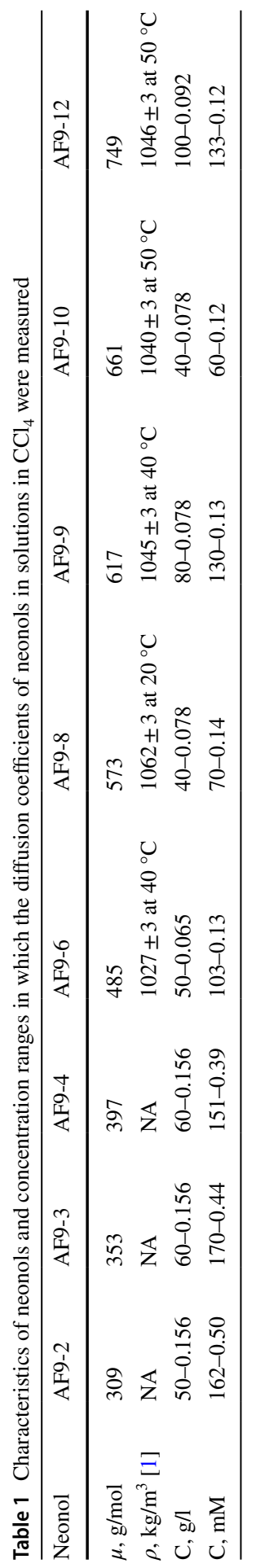




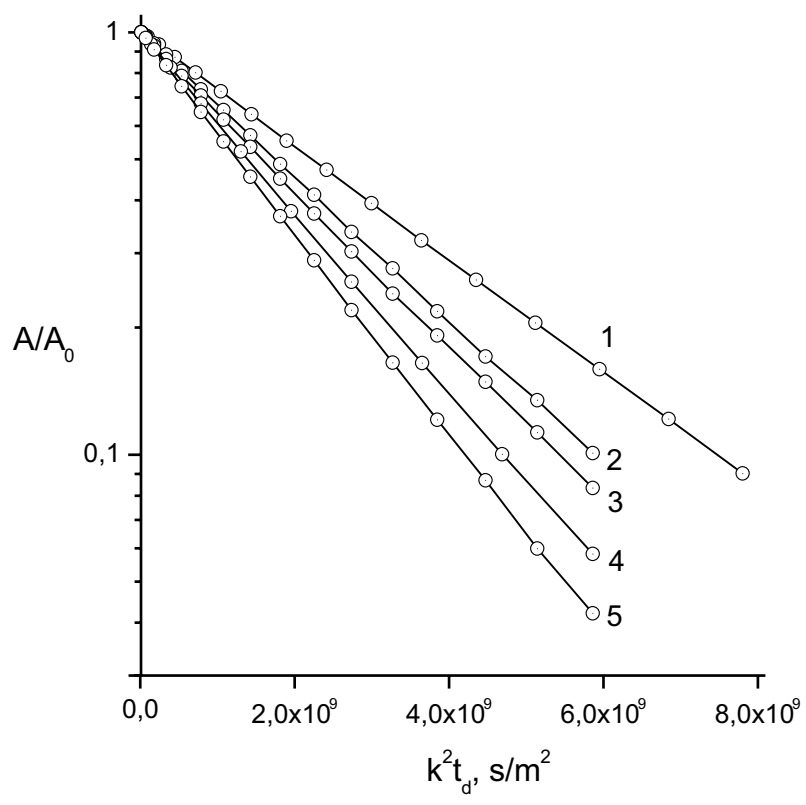

Fig. 1 Diffusion decays for neonols, measured at different concentrations in solutions: 1: AF9-12 (50 g/L), 2: AF9-10 (20 g/L), 3: AF9-6 (3 g/L), 4: AF9-4 (5 g/L), 5: AF9-2 (25 g/L)

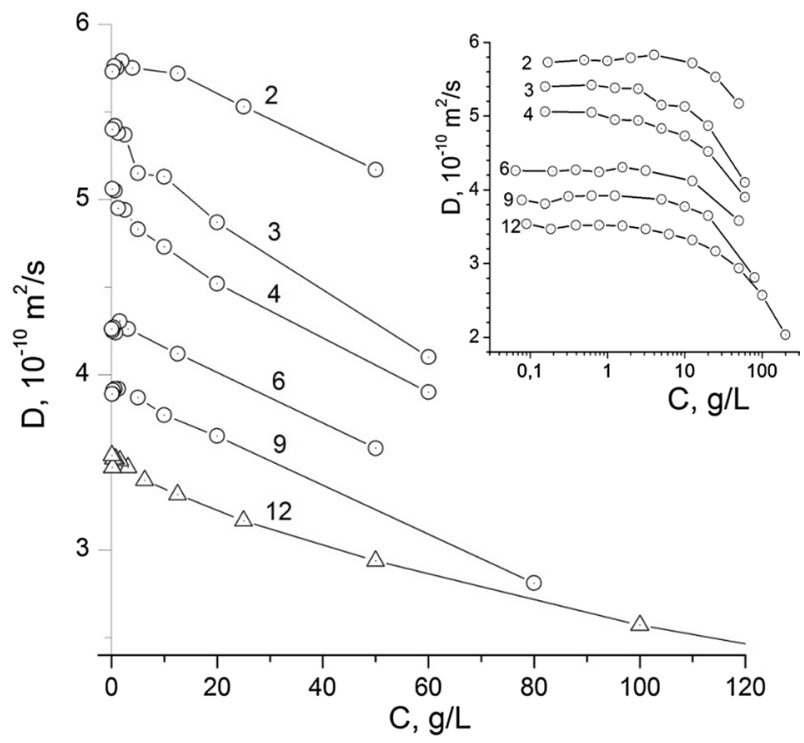

Fig. 2 Diffusion coefficients of neonols AF9-n, depending on their concentration in $\mathrm{CCl}_{4}$. The number of oxyethylene groups in the molecules is shown in the figure. At neonol concentrations of less than $5 \mathrm{~g} / \mathrm{L}$, the infinite dilution condition is satisfied and the diffusion coefficients do not depend on the concentration 
Fig. 3 Diffusion coefficients of neonol molecules depending on the number of oxyethylene groups in the molecules under the condition of infinite dilution

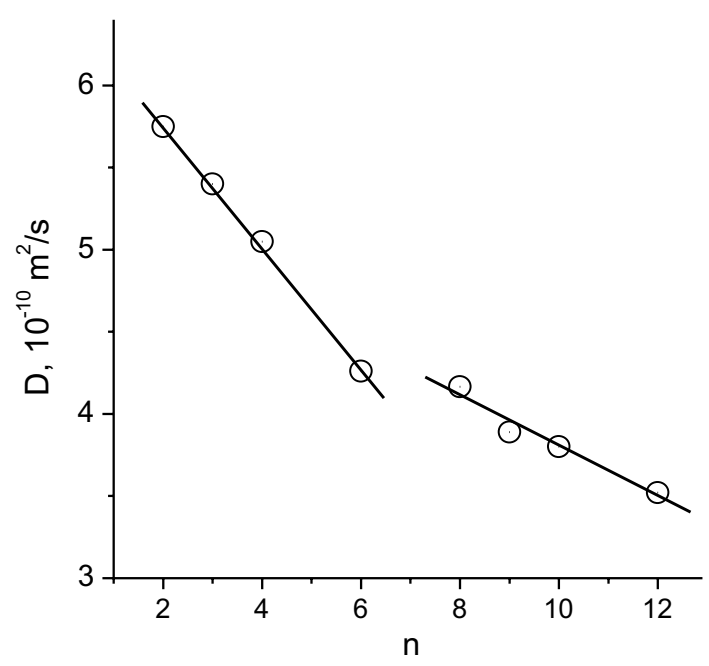

in inclination is associated with a change in the shape of the neonol molecules due to contraction. In the absence of contraction, one would expect a monotonic, linear decrease in $D_{0}$ with increasing $n$.

To estimate the size of the diffusing molecules, the Stokes-Einstein hydrodynamic ratio is often used [28]:

$$
D=\frac{k T}{6 \pi \eta R}
$$

where $k$ is the Boltzmann constant; $T$ is the temperature; and $\eta$ is the coefficient of dynamic viscosity of solvent. The relation (2) obtained for a macroscopic spherical particle in a continuous medium is also used at the molecular level (self-diffusion of molecules in liquids) with corrections for the nature of the interaction of the diffusing particle and solvent [29], taking into account the shape of the particles or diffusing molecules [28]. The shape of the neonol molecules is far from spherical and the sizes of the neonol molecules are of the same order of magnitude as the solvent molecules. Therefore, calculations using relation (2) give a certain effective hydrodynamic radius. With regard to the phenomenon of contraction, it makes sense not to analyze the absolute value of the effective hydrodynamic radius, but its change with increasing length of the oxyethylene chain of neonol molecules.

Using relation (2), we calculated the effective hydrodynamic radii $R$ of the neonol molecules, Fig. 4. In the calculations, the values of $D_{0}$ neonols were used. 
Fig. 4 Effective hydrodynamic radii of neonol molecules, depending on the number of oxyethylene groups in the molecules

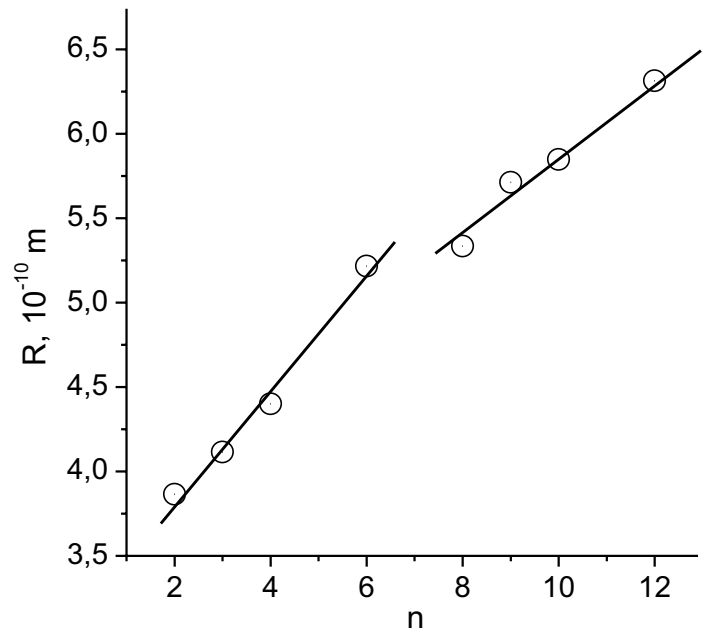

Owing to the infinite dilution, the coefficient of dynamic viscosity of the medium was taken to be equal to the coefficient of viscosity of the solvent, $\mathrm{CCl}_{4}$ [30]. In addition, as shown in Fig. 3, there is a kink in the linear form of the dependence of the hydrodynamic radius $R$ of neonols on the number of oxyethylene groups in the molecules of neonols. The previously performed estimates of the hydrodynamic radii of molecules in pure neonols [17] agree well with the values obtained.

Dynamic changes in the structure of oxyethylene chains of neonol molecules illustrate the models generated by the HyperChem software, Fig. 5, confirming the conclusion about the phenomenon of contraction observed in the interval of $n=6-8$. When $n \leq 6$, the oxyethylene chains retain a regular zigzag shape, while at $n \geq 8$ the chains lose stability, deform, and take on a rolled form.

\section{Conclusions}

In this paper, measurements of diffusion coefficients and calculations of the hydrodynamic radii of neonol molecules in carbon tetrachloride depending on the concentration and number of oxyethylene groups in neonol molecules were obtained. Results indicate changes in the oxyethylene chain shape of the neonol molecules from a zigzag to a meandering shape.

Using $\mathrm{CCl}_{4}$ as a solvent allowed us to study the properties of individual molecules of neonols in true molecular solutions. 
(a)

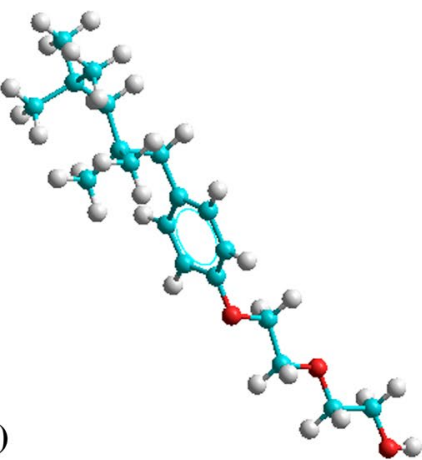

(b)
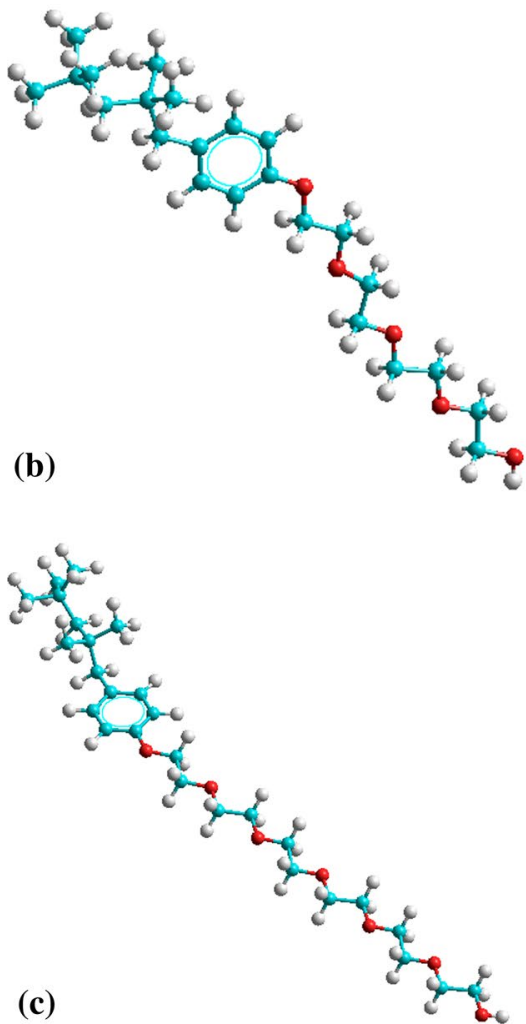
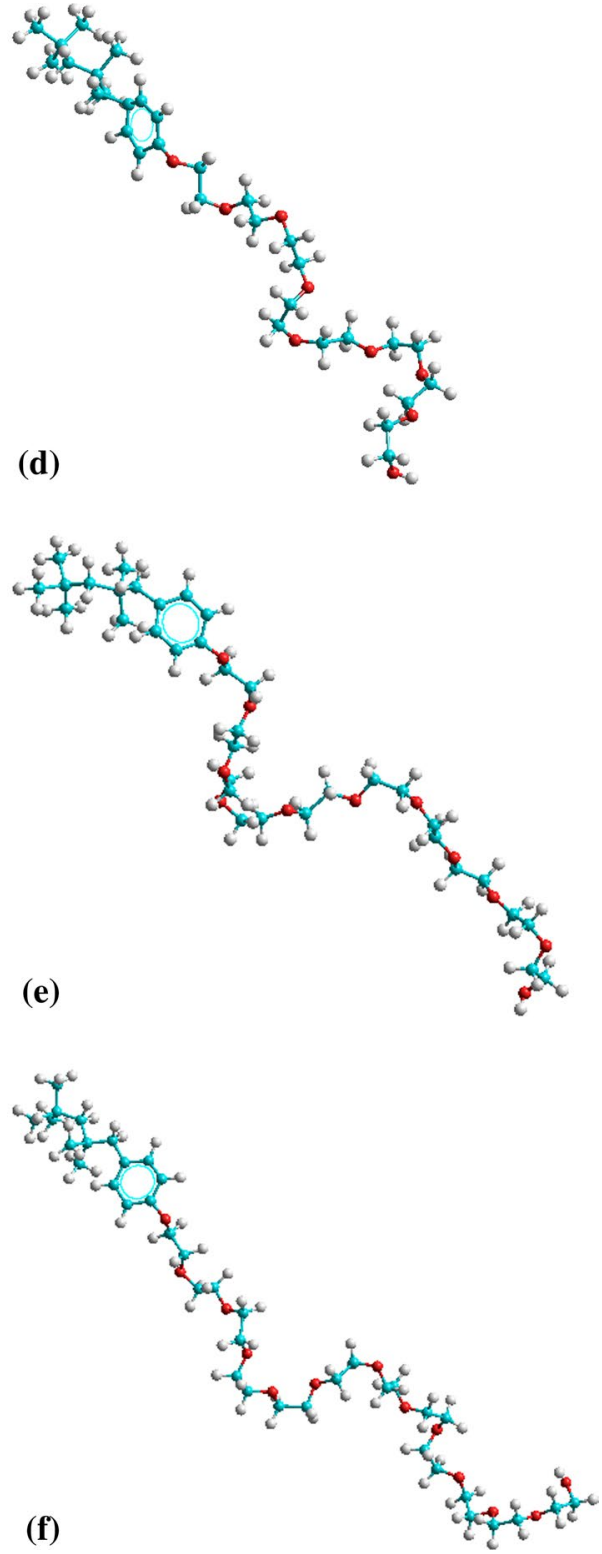

Fig. 5 Models of molecules of neonols: a AF9-2, b AF9-4, c AF9-6, d AF9-8, e AF9-10, f AF9-12, generated using HyperChem 
Acknowledgements Open access funding provided by Lulea University of Technology. "Scriptia Academic Editing" is acknowledged for English correction and proof-reading of this manuscript.

Open Access This article is distributed under the terms of the Creative Commons Attribution 4.0 International License (http://creativecommons.org/licenses/by/4.0/), which permits unrestricted use, distribution, and reproduction in any medium, provided you give appropriate credit to the original author(s) and the source, provide a link to the Creative Commons license, and indicate if changes were made.

\section{References}

1. Nizhnekamskneftekhim Ltd. Neonols. Specifications. Detailed Tables. https://elarum.ru/info/stand ards/tu-2483-077-05766801-98/. Accessed 28 Nov 2018

2. D.I.D. El Eini, B.W. Barry, C.T. Rhodes, J. Coll. Interface Sci. 54, 348 (1976)

3. R.P. Cooney, C.G. Barraclough, T.W. Healy, J. Phys. Chem. 87, 1868 (1983)

4. J.R. Bartlett, R.P. Cooney, J. Chem. Soc., Faraday Trans. 1(82), 597 (1986)

5. T.M. Ferreira, B. Medronho, R.W. Martin, D. Topgaard, Phys. Chem. Chem. Phys. 10, 6033 (2008)

6. A.T. Edwards, A. Javidialesaadi, K. Weigandt, G. Stan, C.D. Eads, https://doi.org/10.26434/chemr xiv.8044235 (2019)

7. P.G. Nilsson, H. Wennerstrom, B. Lindman, J. Phys. Chem. 87, 1377 (1983)

8. J.C. Ravey, M. Buzier, C. Picot, J. Coll. Interface Sci. 97, 9 (1984)

9. V. Rebbin, A. Rothkirch, N. Ohta, T. Hikima, S.S. Funari, Langmuir 30, 1900 (2014)

10. E. Altuntas, U.S. Schubert, Anal. Chim. Acta 808, 56-69 (2013)

11. A.T. Jackson, J.H. Scrivens, J.P. Williams, E.S. Baker, J. Gidden, M.T. Bower, Intern. J. Mass Spectrometry 238, 287 (2004)

12. H.L. Ling, C. Yunnf, K.M. Lai, H.M. Chen, Coll. Surf. A: Phys. Chem. Eng. Aspects 485, 118 (2015)

13. S.P. Gejji, J. Tegenfeldt, J. Lindgren, Chem. Phys. Lett. 226, 427 (1994)

14. C. Jianu, Ethylene Oxide Homogeneous Heterobifunctional Acyclic Oligomers. http://dx.doi. org $/ 10.5772 / 57610$

15. N. Shenfeld, Poverhnostno-Aktivnye Veshestva na Osnove Oksida Etilena (Surface-Active Substances Based on Ethylene Oxide) (Khimiya, Moscow, 1982). (In Russian)

16. V.P. Barabanov, S.A. Bogdanova, Bull. Kazan Chem. Tech. Univ. 4, 7 (2010). (In Russian)

17. V.P. Arkhipov, S.A. Bogdanova, ZSh Idiyatullin, I.V. Lunev, A.V. Filippov, Mendeleev Commun. 26, 355 (2016)

18. E.C. Wijaya, F. Separovic, C.J. Drummond, T.L. Greaves, Phys. Chem. Chem. Phys. 18, 24377 (2016)

19. T.E. Sandoval, M.P. Garate, A.F. Olea, Coll. Surf. A: Phys. Chem. Eng. Aspects 441, 211 (2014)

20. G.N. Smith, P. Brown, C. James, S. E. Rogers, J. Eastoe. http://dx.doi.org/doi:10.1016/j. colsurfa.2016.01.020

21. L.K. Shrestha, R.G. Shrestha, K. Aramaki, J.P. Hill, K. Ariga, Coll. Surf. A: Phys-Chem. Eng. Aspects 414, 140 (2012)

22. J.A. Garate, M.A. Valenzuela, M.P. Garate, A.F. Olea, J. Surf. Deterg. 12, 231 (2009)

23. A.J. Gordon, R.A. Ford, The chemist's companion. A handbook of practical data, techniques, and references (Wiley, New York, 1972)

24. J.E. Tanner, J. Chem. Phys. 52, 2523 (1970)

25. R. Dong, J. Hao, Chem. Rev. 110, 4978 (2010)

26. G.N. Smith, P. Brown, S.E. Rogers, J. Eastoe, Langmuir 29, 3252 (2013)

27. E.C. Voutsas, M.V. Flores, N. Spiliotis, G. Bell, P.J. Halling, D.P. Tassios, Ind. Eng. Chem. Res. 40, $2362(2001)$

28. J.T. Edward, J. Chem. Educ. 47, 261 (1970)

29. R. Zwanzig, M. Bixon, Phys. Rev. 2, 2005 (1970)

30. N.B. Vargaftik, Spravochnik po Teplofizicheskim Svojstvam Gazov i Zhidkostej (Handbook of Thermophysical Properties of Gases and Liquids) (Nauka, Moscow, 1972). (In Russian)

Publisher's Note Springer Nature remains neutral with regard to jurisdictional claims in published maps and institutional affiliations. 